\title{
Eliminating Gender-Based Bias in Academic Medicine: More Than Naming the "Elephant in the Room"
}

\author{
Anna U. Morgan, MD, MSc, MSHP 1,2, Krisda H. Chaiyachati, MD, MPH, MSHP $2,3,4$, \\ Gary E. Weissman, MD, MSHP $2,5,6$, and Joshua M. Liao, MD, MSc $c^{2,7,8}$
}

\begin{abstract}
'Division of General Internal Medicine, Department of Medicine, Harbor-UCLA Medical Center, Torrance, CA, USA; ${ }^{2}$ Leonard Davis Institute of Health Economics, University of Pennsylvania, Philadelphia, PA, USA; ${ }^{3}$ Division of General Internal Medicine, Department of Medicine, Perelman School of Medicine, University of Pennsylvania, Philadelphia, PA, USA; ${ }^{4} \mathrm{Cpl}$. Michael J. Crescenz Veterans Affairs Medical Center, Philadelphia, PA, USA; ${ }^{5}$ Division of Pulmonary, Allergy, and Critical Care Medicine, Department of Medicine, Hospital of the University of Pennsylvania, Philadelphia, PA, USA; ${ }^{6}$ Palliative and Advanced Illness Research Center, Perelman School of Medicine, University of Pennsylvania, Philadelphia, PA, USA; ${ }^{7}$ Division of General Internal Medicine, Department of Medicine, University of Washington, Seattle, WA, USA; ${ }^{8}$ UW Medicine Center for Scholarship in Patient Care Quality and Safety, University of Washington, Seattle, WA, USA.
\end{abstract}

Gender-based discrimination and bias are widespread in professional settings, including academic medicine. Overt manifestations such as sexual harassment have long been identified but attention is only more recently turning towards subtler forms of bias, including inequity in promotion and compensation. Barriers to progress vary across institutions and include lack of awareness, inadequate training, poor informational transparency, and challenging power dynamics. We propose five solutions that the academic medical community can adopt to not only name, but also address, gender-based bias as the proverbial elephant in the room: definitively identify the systemic nature of the problem, prompt those with influence and power to advance a culture of equity, broadly incorporate evidence-based explicit anti-sexist training, increase transparency of information related to professional development and compensation, and use robust research methods to study the drivers and potential solutions of gender inequity within academic medicine. While implementing these proposals is no small task, doing so is an important step in helping the academic medical community become more just.

KEY WORDS: workforce; medical culture; professionalism; physician behavior.

$\mathrm{J}$ Gen Intern Med 33(6):966-8

DOI: $10.1007 / \mathrm{s} 11606-018-4411-0$

(c) Society of General Internal Medicine 2018

$\mathrm{M}$ anifestations of gender-based bias against women, including sexual harassment, discrimination, and inequitable treatment, are common in professional settings. Given recent headlines from the film industry and political

Received June 13, 2017

Revised November 29, 2017

Accepted March 8, 2018

Published online March 21, 2018 community, as well as the robust and powerful \#MeToo movement, it should not surprise us that half of women report gender-based harassment in the American workplace overall. ${ }^{1}$ Women in academic medicine are no exception. Approximately $50 \%$ of female faculty report having experienced sexual harassment and 47-70\% report gender-based discrimination, ${ }^{2}$ while $66 \%$ of physician mothers report experiencing genderbased discrimination. $^{3}$

Beyond being victims of harassment and overt discrimination, women in academic medicine can also experience subtler manifestations of gender-based bias (e.g., disparity in professional promotion and/or salary). For example, since 1995, the number of women and men enrolling in US medical schools has been equivalent. Yet as of 2014, women made up only $34 \%$ of associate professors, $21 \%$ of full professors, $12 \%$ of department chairs in internal medicine, and $16 \%$ of deans at US medical schools. ${ }^{4}$ Nearly two thirds of female clinicianinvestigators endorse personally experiencing gender bias in professional advancement, ${ }^{5}$ and female physicians at US public medical schools earn nearly $\$ 20,000$ less per year than their male counterparts. ${ }^{6}$

Numerous initiatives have arisen to counteract these trends and support the needs of female physicians. For example, the Society for General Internal Medicine has implemented the Women and Medicine Taskforce, ${ }^{7}$ the American Association of Medical Colleges coordinates the Group on Women in Medicine and Science, ${ }^{8}$ and institutions have initiated local programs for female physicians. ${ }^{9}$

Unfortunately, despite growing recognition of gender-based bias as a proverbial "elephant in the room," progress towards equality has been slow. Barriers vary across institutions and include lack of awareness, inadequate anti-bias training, poor informational transparency related to promotion and compensation, and challenging power dynamics. In view of these challenges alongside persistent gender-based bias against women, we believe there is an opportunity for the academic medical community to organize behind more unified solutions to such disparities. 


\section{POTENTIAL SOLUTIONS}

Efforts to directly counteract gender-based bias, in both its implicit forms as well as its overt manifestations, are critical for achieving gender equality in academic medicine. Building on a framework for addressing gender inequity within academic medicine ${ }^{10}$ and drawing from our experiences as junior members of the academic medical community, we propose solutions focused on addressing unconscious gender bias with the hope that they can help achieve gender equity in our community.

First, we can only begin to address problems of inequity by definitively naming the elephant in the room: the systemic nature of gender-based bias. While insufficient in and of itself for affecting change, this kind of clarity represents the first step in creating space for women and men of all training levels to recognize and call out both overt and subtle manifestations of inequity as they occur, by publicly identifying it in the moment, anonymously reporting, or discussing it with involved parties after the event.

Second, those in positions of power within academic medicine - many of whom are men-can unequivocally support efforts that attempt to counteract manifestations of gender-based inequity. We all have roles to play in local transformation within our institutions. However, individuals who have historically wielded power through formal leadership positions and interpersonal dynamics must in many cases play a central role in addressing gender inequity. These efforts could include involvement in institutional policy changes (e.g., required gender parity on committees, targeted proportion of women as grand rounds speakers), role modeling in daily interactions with colleagues, and other forms of support (e.g., sponsoring promotion or advancement of female junior faculty). Because it can be as or more powerful than strategy in dictating behavior, the culture cultivated by those in positions of power will likely influence how comfortable individuals feel speaking up about and addressing gender-based bias.

Third, we should actively counteract bias by incorporating explicit evidence-based anti-sexist training for individuals at all levels within academic medicine, from medical students through senior faculty. Importantly, such trainings would not only focus on removing pre-existing biases, but also impart the skills necessary to oppose social and cultural norms that perpetuate inequity. While not yet widely implemented, programs tested in a variety of academic settings have begun producing improvements in implicit bias, individual behavior, and departmental climate. ${ }^{11,12}$ To have sustainable and widespread impact, high-quality training should be rigorously tested across different institutions and incorporated into processes like maintenance of certification and tenure review.

Fourth, institutions and departments could better address manifestations of inequity through increased transparency and tracking of information about hiring and academic advancement by gender. Specifically, institutions could develop forums (e.g., working groups comprised of diverse stakeholders, "town hall" style meetings) to discuss how to make the academic work environment more receptive for women. Such work could also take advantage of existing inter-professional education, which provides an opportunity to address bias in contexts that extend beyond individual departments or schools. ${ }^{13}$

Finally, because we can only change what we measure, researchers should utilize established conceptual frameworks and methods to study both the internal drivers and external manifestations of gender-based bias within our profession. Despite robust research infrastructure for studying discrimination and disparity for patients, academic medicine is only beginning to apply such rigor to genderbased bias within its own halls. To this end, approaches based on critical gender theory that have been used successfully in other professional settings could be prominently featured in future research. Practitioner inquiry methods, for example, afford researchers the opportunity to study existing power structures and to understand their own role within those structures. ${ }^{14}$ Dedicated funding mechanisms are necessary to support self-reflective and actionable research to combat gender inequity.

We recognize that implementing these solutions is no small task and must be coordinated with efforts aimed at reducing inequity among colleagues from other historically marginalized groups, including those in sexual or racial/ethnic minority groups and disabled communities, among others. The proposed solutions represent starting points for not only naming, but also actively addressing, gender-based bias in order to help the academic medical community become more just.

\section{CONCLUSION}

Like those in other professional fields, women in academic medicine can experience inequity arising from gender-based bias. Despite mounting empiric and anecdotal evidence about the systemic nature of this bias and its external manifestations, more unified strategies and responses are needed. Several solutions - including active sponsorship by those in positions of power, explicit anti-sexist training, increased transparency about hiring and professional advancement, and rigorous research investigation - can help the academic medical community not only name, but also actively address, gender-based bias.

Acknowledgements: The authors would like to thank Dr. Katrina A. Armstrong and Dr. Judy A. Shea for their review of earlier versions of this manuscript for which they were not financially compensated.

Corresponding Author: Anna U. Morgan, MD, MSc, MSHP; Division of General Internal Medicine, Department of Medicine Harbor-UCLA Medical Center, Torrance, CA, USA (e-mail: amorgan@dhs.lacounty. gov).

\section{Compliance with Ethical Standards:}

Conflict of Interest: The authors declare that they do not have a conflict of interest. 


\section{REFERENCES}

1. Report of Co-Chairs Chai R. Feldblum \& Victoria A. Lipnic. Report of the Co-Chairs of the EEOC Select Task Force on the Study of Harassment in the Workplace.; 2016. https://www.eeoc.gov/eeoc/task_force/harassment/upload/report.pdf. Accessed 14 Feb 2018.

2. Carr PL, Ash AS, Friedman RH, et al. Faculty Perceptions of Gender Discrimination and Sexual Harassment in Academic Medicine. Ann Intern Med. 2000;132(11):889. https://doi.org/10.7326/0003-4819132-11-200006060-00007.

3. Adesoye T, Mangurian C, Choo EK, Girgis C, Sabry-Elnaggar H, Linos E. Perceived Discrimination Experienced by Physician Mothers and Desired Workplace Changes. JAMA Intern Med. 2017;31(9):1004-1010. https://doi.org/10.1001/jamainternmed.2017.1394.

4. Lautenberger DM, Dandar VM, Raexer CL, Sloane RA. The state of women in academic medicine: the pipeline and pathway to leadership 2013-2014. Vol 1. 2014. https://members.aamc.org/eweb/upload/The State of Women in Academic Medicine 2013-2014 FINAL.pdf. Accessed $14 \mathrm{Feb} 2018$.

5. Jagsi R, Griffith KA, Jones R, Perumalswami CR, Ubel P, Stewart A. Sexual Harassment and Discrimination Experiences of Academic Medical Faculty. JAMA. 2016;315(19):2120. https://doi.org/10.1001/jama. 2016.2188 .

6. Jena AB, Olenski AR, Blumenthal DM. Sex Differences in Physician Salary in US Public Medical Schools. JAMA Intern Med. 2016;176(9): 1294. https://doi.org/10.1001/jamainternmed.2016.3284.
7. Women and Medicine Task Force. Soc Gen Intern Med. 2018. http:// www.sgim.org/communities/task-forces/women-and-medicine. Accessed 14 Feb 2018.

8. Group on Women in Medicine and Science (GWIMS) - Member Center AAMC. Am Assoc Med Coll. 2018. https://www.aamc.org/members/ gwims/. Accessed 14 Feb 2018.

9. Focus on Health \&amp; Leadership for Women. Trust Univ Pennsylvania. 2018. https://www.med.upenn.edu/focus/. Accessed 14 Feb 2018

10. Westring A, McDonald JM, Carr P, Grisso JA. An Integrated Framework for Gender Equity in Academic Medicine. Acad Med. 2016;91(8):10411044. https://doi.org/10.1097/ACM.0000000000001275.

11. Girod S, Fassiotto M, Grewal D, et al. Reducing Implicit Gender Leadership Bias in Academic Medicine With an Educational Intervention. Acad Med. 2016;91(8):1143-1150. https://doi.org/10.1097/ACM. 0000000000001099.

12. Carnes M, Bartels CM, Kaatz A, Kolehmainen C. Why is John More Likely to Become Department Chair Than Jennifer? Trans Am Clin Climatol Assoc. 2015;126:197-214

13. IPE Teaching FellowsProgram | Center for Health Sciences Interprofessional Education, Research and Practice. https://collaborate.uw.edu/ 2017/07/ipe-teaching-fellows-program/. Accessed 14 Feb 2018.

14. Cochran-Smith $\mathbf{M}$, Lytle $\mathbf{S}$, eds. Inquiry As Stance: Practitioner Research for the Next Generation. New York: Teacher's College Press; 2009. 